\title{
Progressive loss of endothelial P-selectin expression with increasing malignancy in colorectal cancer
}

\author{
Charlotte FJM Peeters ${ }^{1,2}$, Theo JM Ruers ${ }^{2}$, Johan R Westphal ${ }^{1}$ and Robert MW de Waal ${ }^{1}$ \\ ${ }^{1}$ Department of Pathology, University Medical Centre Nijmegen, The Netherlands and ${ }^{2}$ Department of \\ Surgical Oncology, University Medical Centre Nijmegen, The Netherlands
}

\begin{abstract}
Adhesion of inflammatory cells to vascular endothelium is mediated by specific cell adhesion receptors on both leukocytes and endothelial cells. One of the adhesion molecules on the endothelium is P-selectin. Decreased vascular P-selectin expression has been associated with tumor progression in melanoma patients. We now report on the expression of endothelial P-selectin in colorectal cancer (CRC). We studied a colorectal tissue specimen series ranging from normal colorectal tissue via unmetastasized primary tumors to tumors with the same depth of invasion at the primary site but with liver metastases. Moreover, P-selectin expression levels in liver metastases were determined. The number of P-selectin positive vessels as a fraction of the total number of vessels, both intra- and peritumorally, was determined by staining for CD62P and CD34, respectively. Furthermore, by immunostaining for leukocytes (CD45) and macrophages (CD68), it was evaluated whether levels of P-selectin expression influenced infiltrate density and composition. The results showed that levels of peritumoral P-selectin expression were reciprocal to the degree of progression in CRC. This relation was even more pronounced intratumorally: in metastasized primary tumors and in the metastatic lesions, P-selectin expression was virtually absent. This distribution pattern was reflected in the numbers of leukocytes that accumulated in the various tissues, since in the primary tumors with metastases, and in the metastatic lesions, hardly any infiltrating cells were observed. In these lesions, leukocytes were present in the peritumoral zone, but seemed unable to enter the tumor tissue. In primary tumors without metastasis, the intratumoral leukocyte infiltration density was significantly higher. Recruitment levels of macrophages remained constant throughout the different tissues. We suggest that downregulation of endothelial P-selectin expression is a mechanism by which CRC lesions evade inflammatory regression and, thereby, progress to a more advanced stage of malignancy.
\end{abstract}

Laboratory Investigation (2005) 85, 248-256, advance online publication, 10 January 2005; doi:10.1038/labinvest.3700217

Keywords: P-selectin; endothelial cells; colorectal cancer; liver metastasis; leukocytes

Colorectal cancer (CRC) is one of the most common malignancies in the Western world. ${ }^{1}$ Factors associated with the outcome of CRC, such as angiogenesis and leukocyte infiltration, have been studied extensively. ${ }^{2-5}$ Leukocyte infiltration is initiated by extravasation of leukocytes as the result of an appropriate expression of cell surface adhesion receptors both on leukocytes and endothelial cells. Firm adherence of leukocytes to microvascular endothelial cells finally results in diapedesis of leukocytes across the endothelial lining and pene-

Correspondence: Dr CFJM Peeters, MD, Department of Pathology 437, University Medical Centre Nijmegen, PO Box 9101, $6500 \mathrm{HB}$ Nijmegen, The Netherlands.

E-mail: c.peeters@pathol.umcn.nl

Received 14 September 2003; revised and accepted 28 October 2004; published online 10 January 2005 tration, in the case of a tumor, into the surrounding malignant tissue. ${ }^{6-9}$

Among endothelial cell adhesion molecules, the selectin family plays a crucial role in the adhesion of leukocytes to activated endothelium. ${ }^{7,10,11}$ An important member of this family is P-selectin. P-selectin promotes immediate weak interaction and rolling of leukocytes along the vascular endothelium. ${ }^{12}$ In the human melanoma progression series including benign, malignant and metastatic lesions, we demonstrated that the degree of vascular P-selectin expression is inversely related to the level of progression. ${ }^{13}$ There are indications that in primary colorectal tumors endothelial P-selectin expression may be downregulated as well. ${ }^{14}$ Various other studies have reported expression of adhesion molecules on endothelial cells within other primary human tumors. ${ }^{9,15,16}$ Only a few studies addressed 
the level of expression of these molecules in metastatic lesions. ${ }^{13,17}$ Endothelial P-selectin expression in metastases of colorectal carcinoma has not been investigated at all. In the present study, we investigated P-selectin expression in a series of human colorectal tissue specimens encompassing normal colorectal tissue, primary tumors without metastates to lymph nodes or liver, and colorectal tumor specimens with the same invasion depth at the primary site but with liver metastases. Moreover, $P$-selectin expression levels in liver metastases were determined. Furthermore, we investigated the relation between the expression of P-selectin and the presence of inflammatory cells, both bearing the P-selectin ligand and expressing other adhesion molecules. It has been demonstrated that in CRC, inflammatory cells are predominantly distributed along the invasive margin of the tumor. ${ }^{6,18}$ Accordingly, the relation between P-selectin expression and the number of infiltrating cells was investigated in two different areas of the tumor: both in the invasive margin and within the tumor lesion itself. Our findings indicate that one of the mechanisms by which advanced colorectal lesions evade inflammatory regression is via a decrease of endothelial P-selectin expression.

\section{Materials and methods}

\section{Patients}

After informed consent, 22 patients were included in this study, and divided in the following groups: one group of 11 patients with CRC without metastases in lymph nodes or liver (stage $\mathrm{T}_{3-4}, \mathrm{~N}_{0}, \mathrm{M}_{0}$ ); a second group of 11 patients with a colorectal primary tumor with the same depth of invasion as the first group but with liver metastases (synchronous metastases, stage $\mathrm{T}_{3}, \mathrm{~N}_{0-2}, \mathrm{M}_{1}$ ); and a third group consisting of the metastatic tissue samples from these liver metastases. For comparison of results a fourth group of samples was analyzed consisting of normal colorectal tissue. Patient and tumor characteristics are given in Table 1. Representative tissue samples were taken from the CRC excision specimen and the corresponding liver metastases from patients who underwent surgery at the University Medical Centre Nijmegen, The Netherlands.

Patients did not receive any form of preoperative therapy (radiotherapy/chemotherapy) for the primary tumor and the liver metastases.

Based on conventional histopathological examination of paraffin sections, the pathological stage was determined according to the TNM classification. ${ }^{19}$

\section{Immunohistochemistry}

In all, $4 \mu \mathrm{m}$ paraffin sections of normal colon, primary tumor and liver metastases were semiserially cut.
Table 1 Characteristics of the patients

\begin{tabular}{|c|c|c|}
\hline $\begin{array}{l}\text { Number of } \\
\text { patients }\end{array}$ & $\begin{array}{c}C R C \text { without } \\
\text { metastases }\end{array}$ & $\begin{array}{l}\text { CRC with } \\
\text { metastases }\end{array}$ \\
\hline Total number & 11 & 11 \\
\hline Males & 7 & 4 \\
\hline Females & 4 & 7 \\
\hline \multicolumn{3}{|c|}{ Age at surgery (year) } \\
\hline Range & $47-75$ & $44-83$ \\
\hline Mean & 62 & 60 \\
\hline \multicolumn{3}{|l|}{ PTNM } \\
\hline \multicolumn{3}{|l|}{ Tumor size } \\
\hline $\mathrm{T} 1$ & 0 & 0 \\
\hline $\mathrm{T} 2$ & 0 & 0 \\
\hline $\mathrm{T} 3$ & 9 & 11 \\
\hline $\mathrm{T} 4$ & 2 & 0 \\
\hline \multicolumn{3}{|l|}{ Nodal status } \\
\hline No & 11 & 2 \\
\hline $\mathrm{N} 1-2$ & 0 & 9 \\
\hline \multicolumn{3}{|l|}{ Metastatic disease } \\
\hline Mo & 11 & 0 \\
\hline M1 & 0 & 11 \\
\hline \multicolumn{3}{|l|}{ Grade } \\
\hline Well/moderate & 11 & 11 \\
\hline Poor & 0 & 0 \\
\hline
\end{tabular}

After air-drying overnight at $37^{\circ} \mathrm{C}$, sections were deparaffinized in xylol and rehydrated in an alcohol gradient. Endogenous peroxidase activity was quenched by incubation with $0.01 \% \mathrm{H}_{2} \mathrm{O}_{2}$ for 30 min followed by PBS washes. After endogenous avidin and biotin had been blocked in the liver metastases, sections were treated with pronase $0.1 \%$ at $37^{\circ} \mathrm{C}$ for $10 \mathrm{~min}$ and washed in PBS. After preincubation with PBS containing 20\% normal goat serum, sections were incubated with polyclonal rabbit anti-human P-selectin, CD62P (JC/70A, dilution 1:400; Pharmingen, San Diego, CA, USA). Furthermore, sections were incubated with monoclonal antibody against human endothelial antigen CD34 (Qbend/10, dilution 1:750, Neomarkers, Fremont, CA, USA).

Various inflammatory cells were immunostained with the following antibodies: anti-CD45 (antihuman common leukocyte antigen, LCA, dilution 1:160), anti-CD68 (anti-human macrophage, Kpl, dilution 1:100) and anti-elastase (anti-human neutrophil elastase, NP57, dilution 1:800). The latter three antibodies were obtained from Dako A/S, Glostrup, Denmark).

Antibody binding was detected by subsequent incubation with, respectively, biotinylated goat anti-rabbit immunoglobulin (Ig) for P-selectin staining, and biotinylated horse anti-mouse Ig for all other stainings. The alkaline phosphatase complex was detected by fast red. Sections were counterstained with hematoxylin and mounted in Imsol. 


\section{Assessment of P-selectin Positive Vessels}

Vascular density in normal colon, primary CRC with and without metastases and in liver metastases was studied in tissue sections that were stained with anti-CD34 monoclonal antibody.

Intratumoral and peritumoral vessel counts were determined in two randomly chosen, nonoverlapping $\times 100$ fields. Results were expressed as the average number of vessels per $\times 100$ field.

The proportion of vessels stained for P-selectin was determined as a fraction of the total number of CD34-positive vessels that was determined in serial tissue sections. Results were scored by two independent observers who were blinded to patients' characteristics, type of therapy and timing of biopsy. In cases where scores differed, slides were reviewed to reach consensus.

\section{Analysis of Inflammatory Cells}

In normal colon, primary CRC and liver metastases, both intratumorally and along the invasive margin, the numbers of leukocytes, macrophages and granulocytes were determined by staining with anti-CD45, anti-CD68 and anti-elastase antibody, respectively. Results were expressed as number of inflammatory cells per $\times 100$ field in serially cut tissue sections.

\section{Statistical Analysis}

Quantitative data were analyzed by independent T-testing. Correlation between the number of leukocytes and the relative $\mathrm{P}$-selectin positivity was analyzed by using Pearson's product moment correlation coefficient. Significance was defined at $P=0.05$.

All statistical analyses were performed with the SPSS statistical software package, version 9.0.0, for Windows (SPSS Benelux bv, Gorinchem, The Netherlands). Data are presented as mean plus and minus standard deviation (s.d.).

\section{Results}

\section{Staining Characteristics}

Vascular density in normal colon, CRC and synchronous liver metastasis was studied in tissue sections that were stained with anti-CD34 antibody.

In contrast to staining of normal colon and normal liver tissue, which showed widely separated vessels with intact vessel wall integrity, vascular staining of tumor tissue in both primary tumor and liver metastasis demonstrated elongated, strongly dilated and often irregularly shaped vessels, sometimes arranged in clusters (Figure 1). This pattern was identical both intratumorally and peritumorally.
Expression of P-selectin in tissue samples of normal colon, CRC and synchronous liver metastasis was determined by immunohistochemical analysis with anti-CD62P antibody. As shown in Figure 1, in normal colorectal tissue, P-selectin was strongly expressed on larger vessels, and delicate expression was observed on microcapillaries. In primary tumor lesions without metastases, the expression level was slightly decreased. In primary tumor lesions with metastases only a small part of the vessels was P-selectin positive, whereas in liver metastases, P-selectin was completely lacking.

\section{Quantitative Analysis of Vascular Density and P-selectin Expression}

Vascular density in both the intratumoral zone and the area along the invasive margin was assessed by counting the number of vessels per $\times 100$ field in tissue sections stained for CD34. In four patients, the diameter of the peritumoral zone of the liver metastases was very narrow or completely absent. Therefore, determining vascular density in a reliable quantitative fashion was not possible there. For this reason, these cases were excluded from analysis of the peritumoral zone.

Vascular densities, both intra- and peritumorally, are shown in Figure 2. In normal colorectal tissue, the mean number \pm s.d. of vessels/field was $112 \pm 67$. In the intratumoral area of the colorectal primary tumor of patients without metastases to lymph nodes or liver, the mean number of vessels $/ \times 100$ field was significantly lower: $60 \pm 36(P=0.035)$. In contrast, in patients with liver metastases, the intratumoral area of the primary tumor had an increase in vascular density of $140 \pm 37$, which was significantly higher than patients without any metastases $(P=0.001)$. In the intratumoral area of the liver metastases, a lower vascular density was found: $79 \pm 36$. A similar pattern was found in the peritumoral areas, which contained $68 \pm 39$ vessels in colorectal primary tumors without metastases, $129 \pm 32$ in the colorectal tumor with synchronous liver metastases $(P=0.001)$ and $95 \pm 37$ in the peritumoral areas of the liver metastases.

The expression of endothelial P-selectin was determined as a fraction of the number of CD34positive vessels in the colorectal specimen series, both intra- and peritumorally (Figure 3). In normal colon, $51 \pm 10 \%$ of the vessels on the average were P-selectin positive whereas nonmetastazised primary tumors contained a significantly lower percentage of $\mathrm{P}$-selectin-positive vessels in the intratumoral area $(31 \pm 19 \%, P=0.006)$. In the peritumoral area of these lesions, the number of P-selectin positive vessels was $51 \pm 14$, which was significantly higher than the number of vessels in the intratumoral area $(P=0.011)$. Interestingly, in primary colorectal tumors of patients with liver metastases, the decrease of P-selectin expression was far more pronounced, 


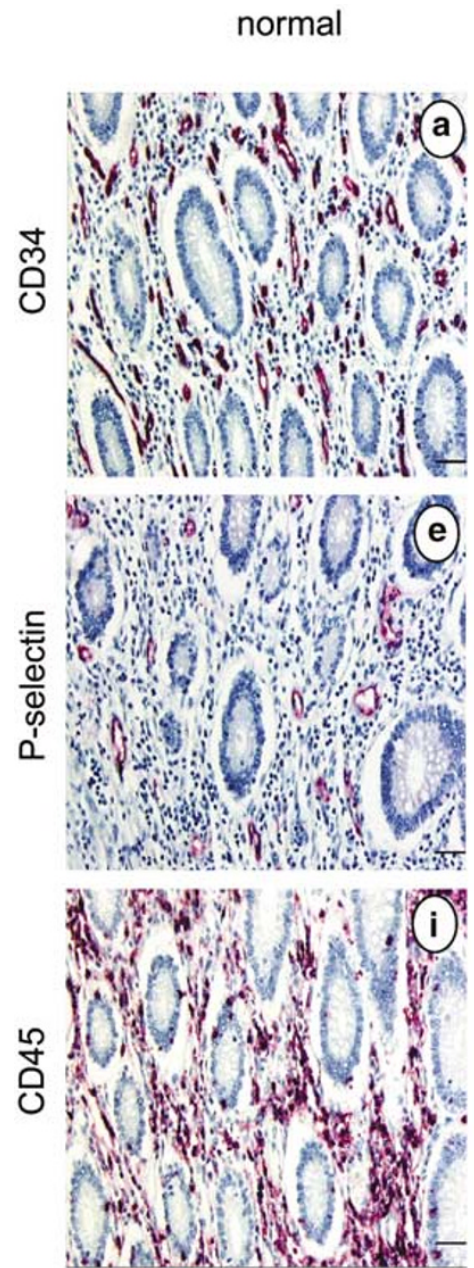

\section{primary tumor without metastases}
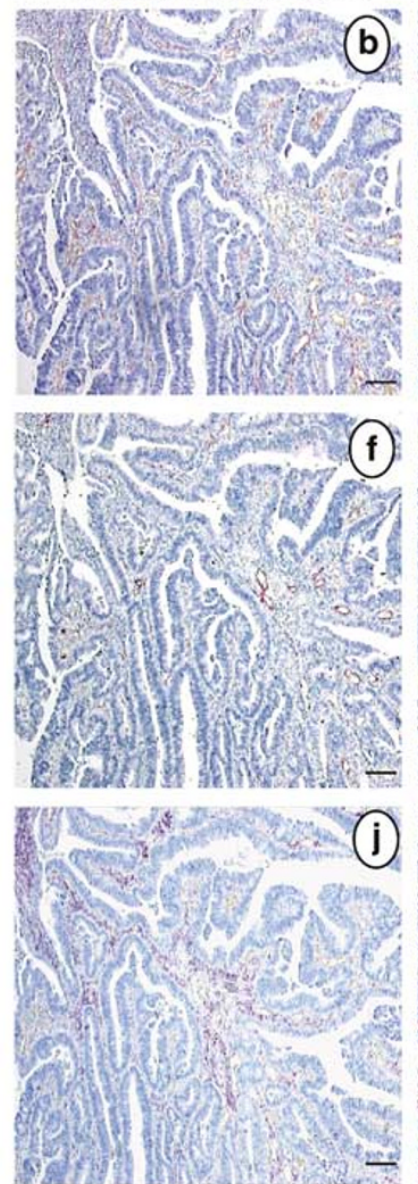

primary tumor with metastases
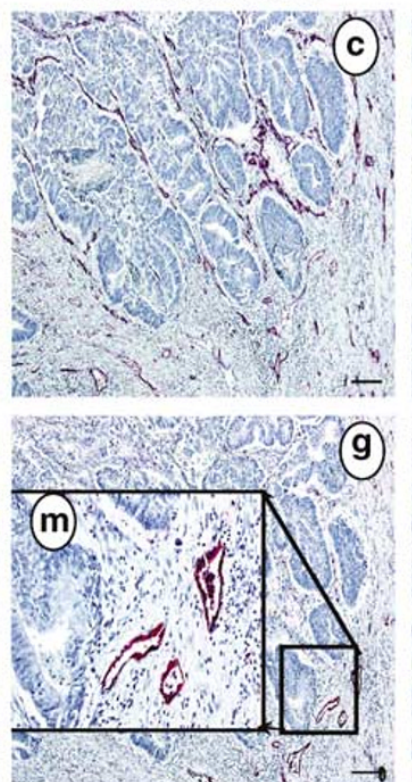

(9)

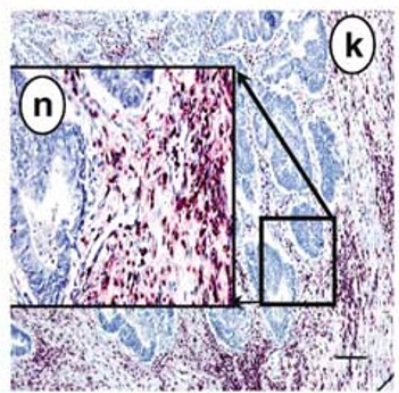

liver metastasis
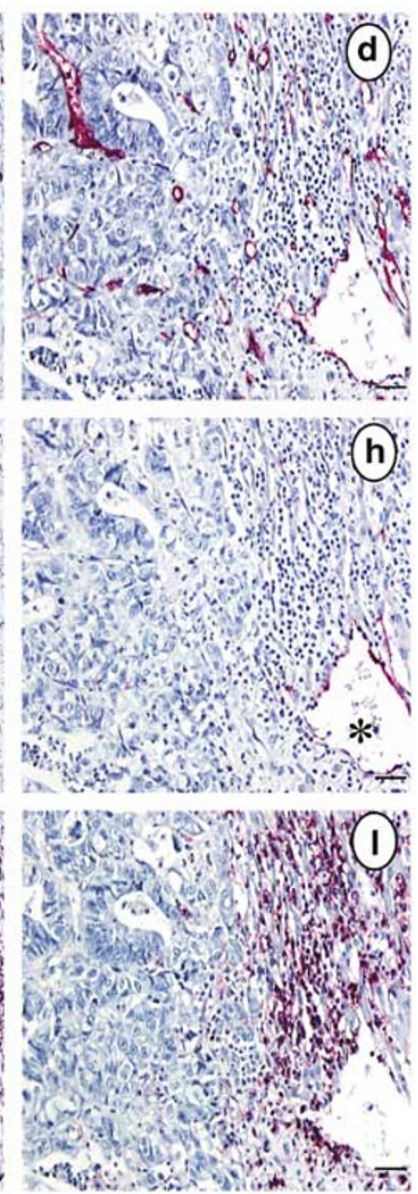

Figure 1 Immunohistochemical analysis of the expression of CD34 (a-d), P-selectin (e-h) and CD45 (leukocytes, i-l) in paraffinembedded sections of serial sections of normal colon (a, e, i), primary CRC without metastases (b, $\mathbf{f}, \mathbf{j})$, primary CRC with metastases (c, $\mathbf{g}$, $\mathbf{k}$ ) and liver metastasis $(\mathbf{d}, \mathbf{h}, \mathbf{l})$. Panels $\mathbf{m}$ and $\mathbf{n}$ show magnifications of parts of $\mathbf{f}$ and $\mathbf{j}$, respectively. In $\mathbf{m}$, P-selectin positive vessels are shown. Panel $\mathbf{n}$ shows leukocyte infiltration around these vessels. Note the absence of P-selectin-positive vessels and leukocytes intratumorally. The asterisk in $\mathbf{h}$ indicates a P-selectin-positive vessel in the peritumoral area of a liver metastasis, with an accumulation of leukocytes as demonstrated in l. Original magnification $\mathbf{b}, \mathbf{f}, \mathbf{j}$ (colorectal primary tumor without metastases) and $\mathbf{c}, \mathbf{g}$, $\mathbf{k}$ (colorectal cancer primary tumor with metastases) $\times 100$, with bar representing $100 \mu \mathrm{m}$. Original magnification $\mathbf{a}, \mathbf{e}, \mathbf{i}$ (normal colon) and d, h and $\mathbf{l}$ (colorectal liver metastasis) $\times 250$, with bar representing $40 \mu \mathrm{m}$.

both intra- and peritumorally: $6.6 \pm 9.6 \%$ and $23.0 \pm$ $17.5 \%$, respectively. Statistical analysis revealed that both in the intratumoral and the peritumoral area this decline was significantly higher than in patients without metastases $(P=0.001$ and 0.001 , respectively). A representative example of the relative absence of P-selectin staining of the tumor vessels within the primary colorectal lesion compared to the vessels in the surrounding tissue is shown in Figure 1.

A similar lack of expression could be demonstrated in liver metastases, with $2.8 \pm 5.0 \%$ of the blood vessels positive for P-selectin in the intratumoral area of the liver metastases $(n=11)$ and $5.9 \pm 9.7 \%$ positive vessels in the perilesional tissue $(n=7)$ (Figure 3).

Perilesional staining for P-selectin in the liver metastases was significantly lower compared to
P-selectin staining of the primary tumor without and with metastases $(P=0.0005$ and 0.033 , respectively). Also, intralesional staining showed a significantly lower amount of P-selectin-positive vessels compared to the primary tumor tissue of patients without metastases $(P=0.001)$. In contrast, compared to primary tumor tissue of patients with synchronous liver metastases intralesional staining did not significantly differ as it was almost absent in the area in both groups.

\section{Distribution of Inflammatory Cells}

Next, the influx of different types of cells involved in the immune response was evaluated in relation to downregulation of endothelial P-selectin. The presence of leukocytes in normal colon tissue, primary 


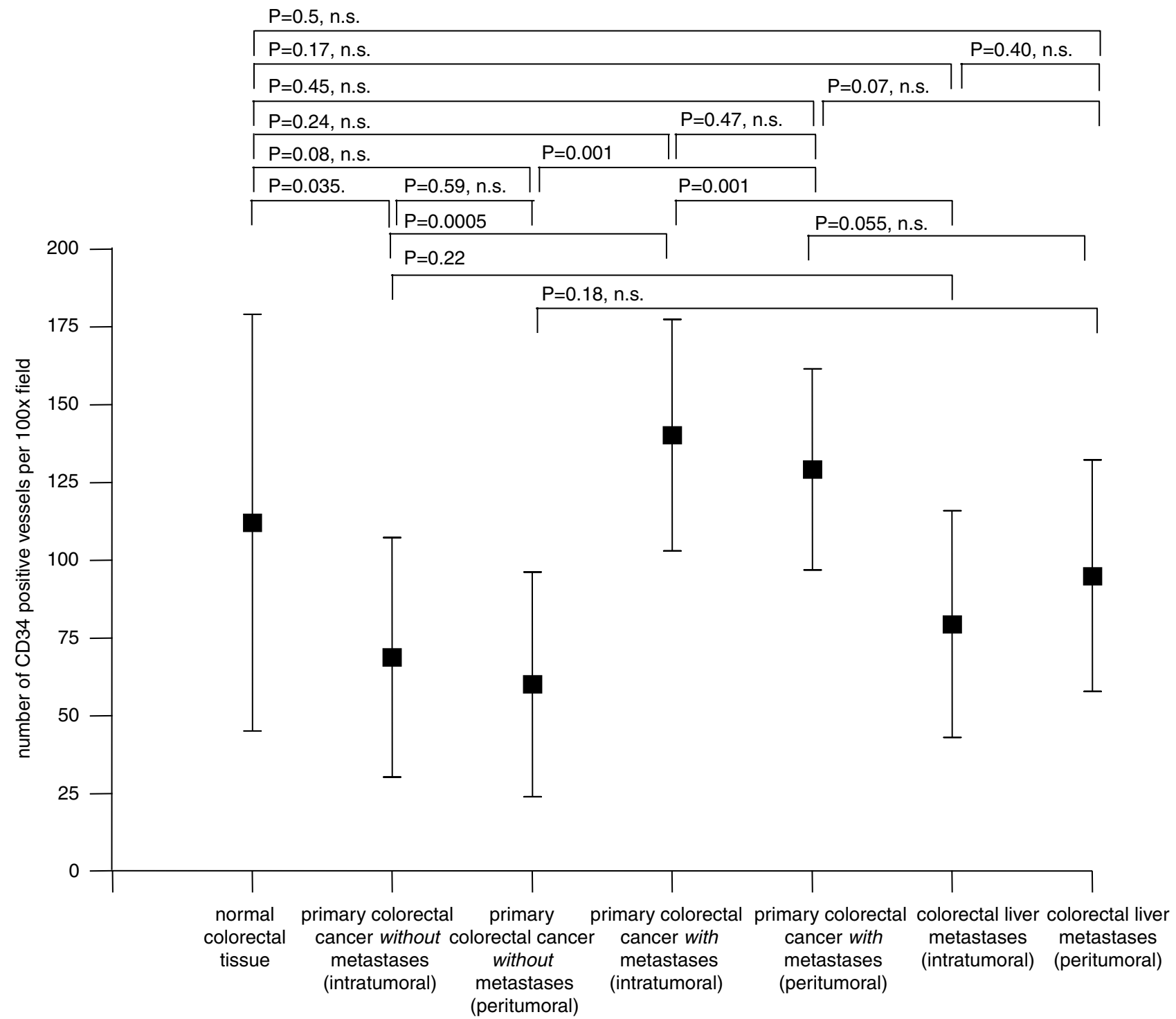

Figure 2 Intratumoral and peritumoral vascular density as determined by counting of CD34 stained vessels in normal colon, colorectal primary tumor without metastases, primary tumor with metastases and liver metastasis. Vascular density is expressed as number of vessels per $\times 100$ field, mean \pm s.d. (mean of the two counted fields for each specimen averaged for the 11 specimens in each group).

tumor (with and without metastases) and liver metastases, both within the tumor and along the peritumoral area, was evaluated by immunohistochemical analysis using CD45, the leukocyte common antigen.

The infiltration density of leukocytes (mean density \pm s.d, per $\times 100$ field) in normal colon was $832 \pm 623$ (Figure 4). In the intratumoral zone of colorectal primary tumors without metastases, the number of leukocytes was significantly lower $(302 \pm 208, P=0.02)$. Interestingly, in the peritumoral area of these lesions, where P-selectin expression was equal to the control group, leukocyte density was higher, and not differed significantly from the control group $(463 \pm 201$ leukocytes/per $\times 100$ field). Moreover, both the intra- and peritumoral area of the colorectal primary tumor of patients with synchronous liver metastases were marked by an even further decrease in leukocyte counts. Again, these differences were more pronounced in the intratumoral area $(122 \pm 192)$ compared to the invasive front of the primary tumor $(496 \pm 369)$. Compared to patients without any lymph or liver metastases, these differences were significantly lower in the intratumoral area $(P=0.049)$ of the tumor. Also, the liver metastases contained generally higher leukocyte count at the invasive front $(477 \pm 294)$ than in the intratumoral areas of these lesions $(108 \pm 243$, $P=0.01$.

Taken together, the observed decrease in leukocyte infiltration in the peri- and intratumoral zone appeared to run parallel to the percentage of $\mathrm{P}$ selectin-positive vessels. 


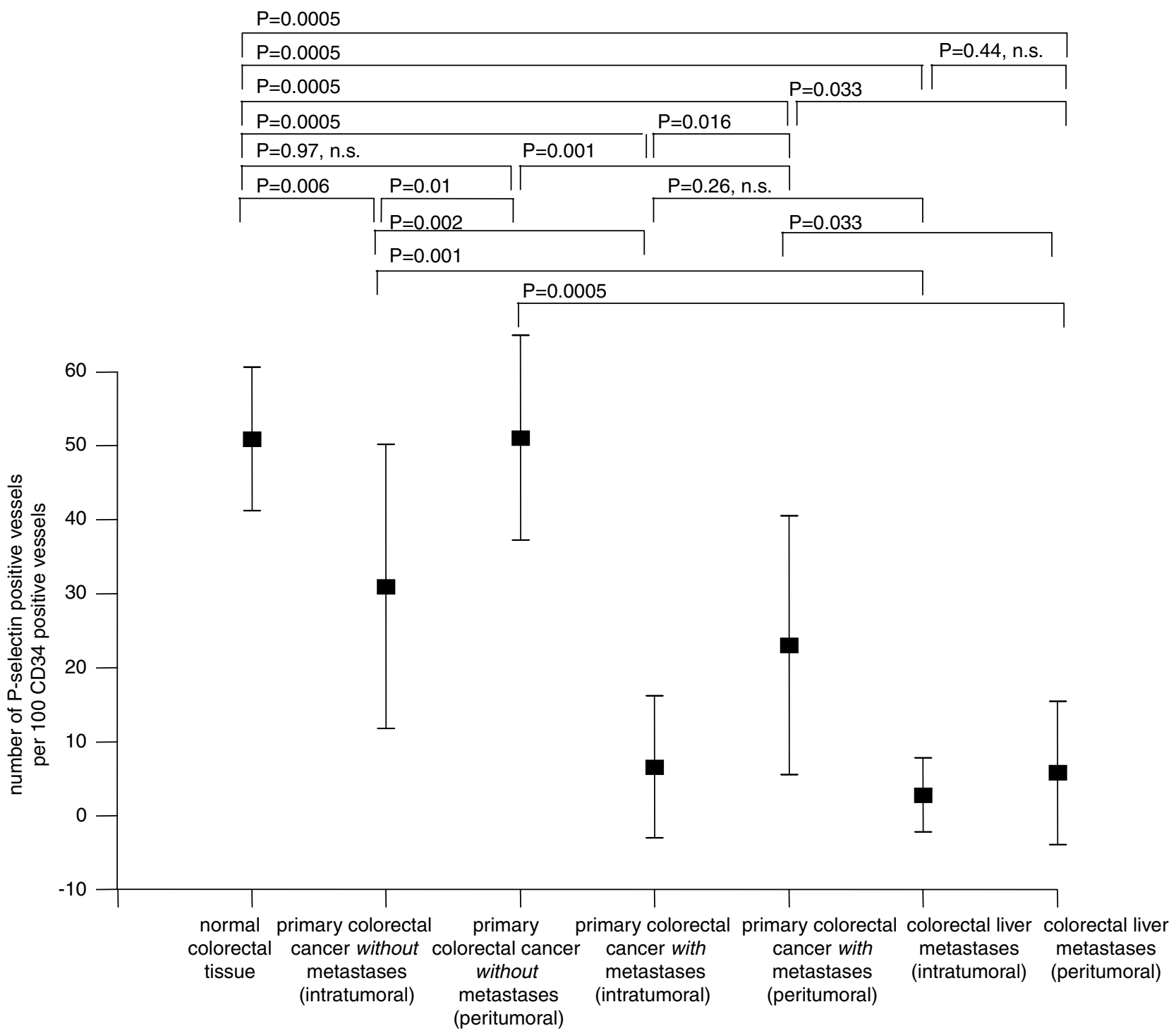

Figure 3 Intratumoral and peritumoral endothelial P-selectin expression in normal colon, primary colorectal tumor (without and with metastases) and liver metastases (number of P-selectin positive vessels per 100 CD $34+$ vessels per $\times 100$ field, mean \pm s.d.; mean of the two counted fields for each specimen averaged for the 11 specimens in each group). Note that levels of peritumoral and intratumoral P-selectin expression is reciprocal to the degree of progression in colorectal cancer.

Correlation analysis between the percentage of P-selectin-positive vessels and the number of leukocytes in the colorectal specimen series revealed a correlation of 0.44 , representing a significant correlation ( $P=0.0005$, Figure 5). A representative example of P-selectin expression and leukocyte colocalization is shown in Figure 1.

The infiltrate was quantitatively analyzed in more detail by counting the number of macrophages and granulocytes per $\times 100$ field.

The mean number \pm s.d. of macrophages per $\times 100$ field in all the 73 specimens analyzed was $223 \pm 249$. Macrophages were equally distributed in the different lesions and no differences between the groups could be demonstrated (data not shown). Granulocyte counts were highly variable both in normal and pathological tissues, and did not allow any conclusions (data not shown).

\section{Discussion}

The purpose of the study reported here was to evaluate the expression of $\mathrm{P}$-selectin (CD62P) on the blood vessel endothelium in CRC. First, we analyzed the vascular density in all tissue specimens, to be able to express the number of P-selectin-positive vessels as a percentage of overall vessel density. Remarkably, a high vessel density was present in primary lesions that had already metastasized, confirming previous publications. ${ }^{20}$ Our subsequent data showed a clear and progressive decrease in the 


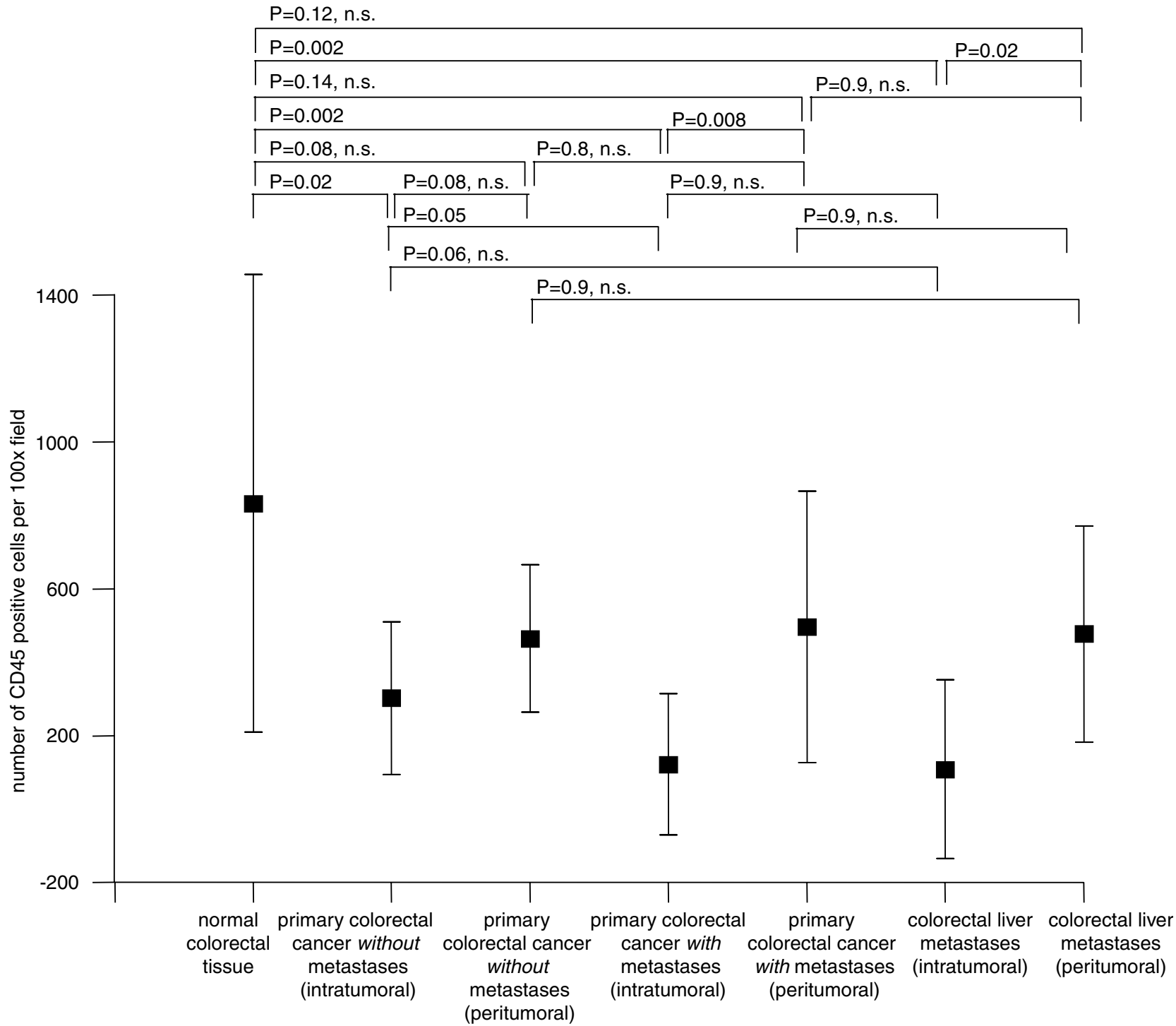

Figure 4 Intratumoral and peritumoral leukocyte density as assessed by CD45 staining of normal colon, primary tumor (without and with metastases) and liver metastases (number of leukocytes per $\times 100$ field, mean \pm s.d.; mean of the two counted fields for each specimen averaged for the 11 specimens in each group). Note a dense distribution of leukocytes along the invasive margin of both the primary tumor and the metastases, whereas the intratumoral area is devoid of leukocytes.

number of P-selectin-positive blood vessels in a series of lesions ranging from normal colon, primary tumors without metastasis to the lymph nodes or liver to primary tumor specimens with comparable depths of invasion at the primary site; but with liver metastases, up to the liver metastases themselves. Similar to what we described earlier in cutaneous melanoma, we were able to demonstrate that the degree of vascular P-selectin expression is reciprocal to the degree of progression in CRC. ${ }^{13}$

Subsequent infiltrate analysis showed a close association between the expression level of $\mathrm{P}$ selectin and the numbers of inflammatory cells. When comparing endothelial P-selectin expression in tissue section of patients without metastases, with lesions with the same invasion depth but with synchronous liver metastases, endothelial P-selectin expression was observed predominantly in the patients without metastases. Moreover, when comparing endothelial P-selectin expression in the invasive margin with that in the intratumoral area of both these types of lesions, a far more pronounced P-selectin expression along the invasive margin was observed. In the peritumoral margin, the most dense leukocyte infiltrates were also present. In contrast, inside the tumor lesion, a lesser percentage of blood vessels usually expressed this endothelial cell adhesion molecule, and fewer leukocytes had accumulated. We encountered these phenotypical variations not only in colorectal primary tumors but also in metastatic lesions which has, to the best of our knowledge, never been described before. Taken these observations together, the following conclusions may be drawn. 


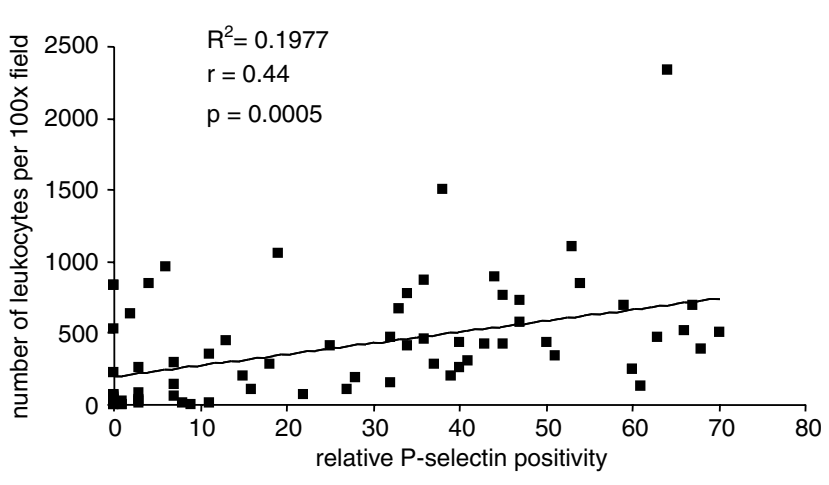

Figure 5 Correlation analysis between the percentage of $\mathrm{P}$ selectin-positive vessels and the number of CD45 + leukocytes in the colorectal cancer tissue specimen series (normal colon, colorectal primary tumor (with and without metastases) and liver metastases). (Pearson's correlation coefficient: $r=0.44$, $P=0.0005)$.

First, our data strongly suggest that there is heterogenecity of colorectal tumor vessels with regard to P-selectin expression: on peritumoral vessels, higher levels were observed. These phenotypical variations were encountered not only in the primary tumors but also in the metastatic lesions. In the peritumoral area of the metastases leukocyte infiltration, however, was more pronounced than that could be expected on the basis of the minor increase in endothelial P-selectin expression. We are currently analyzing the process of vessel formation and remodeling in colorectal liver metastases. Preliminary results demonstrate that in the peritumoral area, a conversion occurs from the wellorganized hepatic sinusoids to an irregularly shaped vascular bed with an upregulation of several endothelial cell molecules, resembling the vasculature in the primary tumor. Therefore, we speculate that the process of leukocyte infiltration into the peritumoral area of liver metastases may be dependent on upregulation of endothelial adhesion receptors other than the selectins.

Next, compared to normal colorectal tissue, we found a downregulation in P-selectin expression and a concomitant decrease in leukocyte infiltration, both in the peritumoral and intratumoral area. Prevention of leukocyte infiltration into the tumor may represent a protective mechanism by which tumors escape immune surveillance. For example, leukocyte infiltration within tumors is generally associated with decreased tumor growth rate in carcinomas of the breast, stomach and colon. ${ }^{18,21-23}$ Our results suggest that in CRC, malignant progression of the tumor leads to progressive P-selectin down regulation. This phenomenon has been described also for other endothelial cell-associated adhesion molecules in colorectal tumors (ICAM-1, E-selectin). ${ }^{14}$

Our results furthermore demonstrate that impaired leukocyte infiltration is not only associated with increased tumor growth, but also with the ability of tumor cells to metastasize. Since the primary tumors that had already metastasized, also had increased vascular density, it is difficult to dissect the mechanisms involved in these processes. Lack of P-selectin expression may result in deficient antitumor immunity, and thereby enhance growth of the primary tumor. On the other hand, a dense vascular bed will augment the tumor's blood supply and stimulate growth as well. It may, however, also facilitate tumor cell invasion and metastasis.

Previously, P-selectin expression and leukocyte infiltration was already related to adverse prognosis in patients with malignant melanoma. ${ }^{24}$ It has been speculated that leukocytes secrete growth factors that stimulate tumor cell proliferation. ${ }^{25} \mathrm{~A}$ downregulation in endothelial P-selectin expression may therefore result in the recruitment of fewer, growth factor producing, leukocytes and in a less growth stimulating microenvironment for the tumor cells. ${ }^{26-}$ ${ }^{28}$ However, our results demonstrate that, compared to patients with liver metastases, in patients without any metastases a high expression level of endothelial P-selectin was maintained, and that this is accompanied by high numbers of infiltrating leukocytes. As tumors without metastases generally have a less infiltrating character and a better prognosis, we suggest that the latter mechanism, that is, tumor growth promotion by infiltrating cells, is less likely to occur. Accordingly, Harrison et $a{ }^{29}$ have demonstrated in patients with right-sided colon cancer, that lymphoid aggregates took precedence as independent prognostic factor for length of survival.

It has been demonstrated that macrophages play a key role in the induction of angiogenesis by the production of vascular endothelial growth factor (VEGF) ${ }^{30}$ In the primary tumor, relatively high levels of endothelial P-selectin were expressed compared to the liver metastases. A decrease in infiltrating macrophages as a result of a downregulation of endothelial P-selectin could therefore explain the observed decrease in angiogenesis in the liver metastases. However, by analyzing the number of infiltrating macrophages, we found no differences between primary tumors and metastases, both intraand peritumorally. Still, the lack of proliferation of the micovasculature in the metastases might be due to a decrease in P-selectin expression, but its mechanism remains unclear. We also speculated that the primary colorectal tumor produces antiangiogenic factors, like angiostatin and endostatin, that inhibit angiogenesis and therefore reduce the number of vessels in its distant metastases, but this remains to be demonstrated in humans.

Taken together, we have demonstrated in a series of CRC lesions that the levels of vascular P-selectin expression are inversely related to the degree of tumor progression. Furthermore, leukocyte infiltration levels showed the same correlation in this series. Our results may have implications for cancer therapy in both the primary CRC and its metastases. Determining the levels of endothelial adhesion 
molecules like P-selectin in tumors may have predictive value for the successfulness of immunotherapy. By influencing the adhesion characteristics of the intratumoral vessels, for example, selectin expression, via cytokine treatment, influx of immuno-competent cells may be encouraged.

\section{References}

1 Wilmink AB. Overview of the epidemiology of colorectal cancer. Dis Colon Rectum 1997;40:483-493.

2 Di Giorgio A, Botti C, Tocchi A, et al. The influence of tumor lymphocytic infiltration on long term survival of surgically treated colorectal cancer patients. Int Surg 1992;77:256-260.

3 Berghella AM, Pellegrini P, Del Beato T, et al. Prognostic significance of immunological evaluation in colorectal cancer. Cancer Biother Radiopharm 1996; 11:355-361.

4 Papamichael D. Prognostic role of angiogenesis in colorectal cancer. Anticancer Res 2001;21:4349-4353.

5 Folkman J. Angiogenesis in cancer, vascular, rheumatoid and other disease. Nat Med 1995;1:27-31.

6 Nash JR. Macrophages in human tumours: an immunohistochemical study. J Pathol 1982;136:72-83.

7 Kansas GS. Selectins and their ligands: current concepts and controversies. Blood 1996;88:3259-3287.

8 Butcher EC. Leukocyte-endothelial cell recognition: three (or more) steps to specificity and diversity. Cell 1991;67:1033-1036.

9 Carlos TM. Leukocyte recruitment at sites of tumor: dissonant orchestration. J Leukoc Biol 2001;70: 171-184.

10 Tedder TF, Steeber DA, Chen A, et al. The selectins: vascular adhesion molecules. FASEB J 1995;9:866873.

11 Vestweber D, Blanks JE. Mechanisms that regulate the function of the selectins and their ligands. Physiol Rev 1999;79:181-213

12 Somers WS, Tang J, Shaw GD, et al. Insights into the molecular basis of leukocyte tethering and rolling revealed by structures of $\mathrm{P}$ - and E-selectin bound to SLe(X) and PSGL-1. Cell 2000;103:467-479.

13 Nooijen PT, Westphal JR, Eggermont AM, et al. Endothelial P-selectin expression is reduced in advanced primary melanoma and melanoma metastasis. Am J Pathol 1998;152:679-682.

14 Suzuki Y, Ohtani H, Mizoi T, et al. Cell adhesion molecule expression by vascular endothelial cells as an immune/inflammatory reaction in human colon carcinoma. Jpn J Cancer Res 1995;86:585-593.

$15 \mathrm{Kuzu}$ I, Bicknell R, Fletcher CD, et al. Expression of adhesion molecules on the endothelium of normal tissue vessels and vascular tumors. Lab Invest 1993; 69:322-428.

16 Ye C, Kiriyama K, Mistuoka C, et al. Expression of E-selectin on endothelial cells of small veins in human colorectal cancer. Int J Cancer 1995;61:455-460.

17 Renkonen J, Paavonen T, Renkonen R. Endothelial and epithelial expression of sialyl Lewis(x) and sialyl Lewis(a) in lesions of breast carcinoma. Int J Cancer 1997;74:296-300.

18 Jass JR, Love SB, Northover JM. A new prognostic classification of rectal cancer. Lancet 1987;1:13031306.

19 Scott-Conner CE, Christie DW. Cancer staging using the American Joint Committee on Cancer TNM System. J Am Coll Surg 1995;181:182-188.

20 Takahashi Y, Bucana CD, Cleary KR, et al. p53, vessel count, and vascular endothelial growth factor expression in human colon cancer. Int J Cancer 1998;79: 34-38.

21 Kreider JW, Bartlett GL, Butkiewicz BL. Relationship of tumor leucocytic infiltration to host defense mechanisms and prognosis. Cancer Metastasis Rev 1984;3: $53-74$.

22 Hamlin IM. Possible host resistance in carcinoma of the breast: a histological study. $\mathrm{Br} \mathrm{J}$ Cancer 1968;22:383-401.

23 Black MM, Freeman C, Mork T, et al. Prognostic significance of microscopic structure of gastric carcinomas and their regional lymph nodes. Cancer 1971;27:703-711.

24 Cochran AJ. Histology and prognosis in malignant melanoma. J Pathol 1969;97:459-468.

25 Kim YJ, Borsig L, Varki NM, et al. P-selectin deficiency attenuates tumor growth and metastasis. Proc Natl Acad Sci USA 1998;95:9325-9330.

26 Reynolds JL, Akhter J, Adams WJ, et al. Histamine content in colorectal cancer. Are there sufficient levels of histamine to affect lymphocyte function? Eur J Surg Oncol 1997;23:224-227.

27 Dabbous MK, North SM, Haney L, et al. Effects of mast cell-macrophage interactions on the production of collagenolytic enzymes by metastatic tumor cells and tumor-derived and stromal fibroblasts. Clin Exp Metastasis 1995;13:33-41.

28 Dimitriadou V, Koutsilieris M. Mast cell-tumor cell interactions: for or against tumour growth and metastasis? Anticancer Res 1997;17:1541-1549.

29 Harrison JC, Dean PJ, el Zeky F, et al. Impact of the Crohn's-like lymphoid reaction on staging of rightsided colon cancer: results of multivariate analysis. Hum Pathol 1995;26:31-38.

30 Xiong M, Elson G, Legarda D, et al. Production of vascular endothelial growth factor by murine macrophages: regulation by hypoxia, lactate, and the inducible nitric oxide synthase pathway. Am J Pathol 1998;153:587-598. 